\title{
Long-chain Polyunsaturated Fatty Acid Supplementation Improves Mood in Elderly Japanese Men
}

\author{
Hisanori Tokuda ${ }^{1 *}$, Toshiaki Sueyasu ${ }^{1}$, Hiroshi Kawashima ${ }^{1}$, Hiroshi Shibata ${ }^{1}$ and \\ Yoshihiko Koga ${ }^{2}$ \\ ${ }^{1}$ Institute for Health Care Science, Suntory Wellness Ltd., 8-1-1 Seikadai, Seika-cho, Soraku-gun, Kyoto 619-0284, JAPAN \\ ${ }^{2}$ Department of Neuropsychiatry, Kyorin University School of Medicine, 6-20-2 Shinkawa, Mitaka, Tokyo 181-8611, JAPAN
}

\begin{abstract}
Although several studies have reported the effects of long-chain polyunsaturated fatty acid (LCPUFA) supplementation on the mood in healthy adults, the effects of LCPUFA on elderly individuals remain unclear. Thus, we hypothesized that LCPUFA supplementation improves mood in the elderly. To address this hypothesis, 115 elderly Japanese men aged 55-64 years were assigned and randomly allocated to the LCPUFA or placebo group. Participants received 4 weeks of supplementation with LCPUFAcontaining oil (docosahexaenoic acid (DHA) $300 \mathrm{mg} / \mathrm{day}$, eicosapentaenoic acid (EPA) $100 \mathrm{mg} / \mathrm{day}$, arachidonic acid (ARA) $120 \mathrm{mg} /$ day) or a placebo oil. Mood was assessed using the Profile of Mood States (POMS) before and after supplementation as the secondary outcome in a previously performed randomized controlled trial on cognitive function. A total of 113 participants completed the supplementation period. One hundred participants (LCPUFA, $n=51$; placebo, $n=49$ ) who were eligible for evaluation of mood were analyzed. Increases in vigor scores on POMS, reflecting a positive mood, were significantly larger in the LCPUFA group than in the placebo group (LCPUFA, +1.8 ; placebo, $-\mathbf{0 . 5}$ ). No significant differences were observed in changes in other negative mood scores between groups. DHA and ARA content in plasma phospholipids were increased by $0.8 \%$ and $0.7 \%$, respectively, in the LCPUFA group, and were significantly larger than those in the placebo group. Dietary DHA, EPA, and ARA intake was unchanged during the study. These results suggest that LCPUFA supplementation may improve vigor (positive mood) in elderly Japanese men.
\end{abstract}

Key words: emotions, arachidonic acid, docosahexaenoic acid, eicosapentaenoic acid, randomized controlled trial

\section{INTRODUCTION}

Recognition of the importance of mental health in the elderly is increasing. Researchers expect to identify factors that affect mood, not only in patients with mental illness but also in healthy elderly individuals. The effects of nonpharmacological treatments, such as exercise or relaxation, have been reported ${ }^{1-4)}$, and dietary constituents are also considered to affect mood in healthy individuals ${ }^{5 \text { ) }}$.

One important nutrient that may affect mood is longchain polyunsaturated fatty acids (LCPUFA), such as docosahexaenoic acid (DHA) and arachidonic acid (ARA), which are present in marine and animal foods and are the major components of brain phospholipids ${ }^{6,7)}$. In animal studies, these LCPUFA are involved in the regulation or maintenance of brain functions such as neural membrane fluidity, synaptic plasticity, and neurogenesis ${ }^{8-14)}$. In epidemiological studies, a relationship between mood and LCPUFA intake, especially DHA and eicosapentaenoic acid(EPA), was reported in mood disorders such as depression ${ }^{15,33,34)}$, and studies examining healthy people have also been recently reported $^{15)}$. These studies show a possible beneficial effect of LCPUFA in depressed individuals, but available data on healthy individuals is still limited.

Profile of Mood States (POMS) was developed as a psychological test for evaluating $\operatorname{mood}^{16)}$. POMS can evaluate

Abbreviations: LCPUFA, long-chain polyunsaturated fatty acids; FA, fatty acid; ARA, arachidonic acid; EPA, eicosapentaenoic acid; DHA, docosahexaenoic acid; POMS, profile of mood states; BDHQ, brief self-administered diet history questionnaire; RCT, randomized controlled trail

\footnotetext{
*Correspondence to: Hisanori Tokuda, Institute for Health Care Science, Suntory Wellness Ltd., 8-1-1 Seikadai, Seika-cho, Sorakugun, Kyoto 619-0284, JAPAN

E-mail: Hisanori_Tokuda@suntory.co.jp

Accepted March 21, 2017 (received for review February 9, 2017)

Journal of Oleo Science ISSN 1345-8957 print / ISSN 1347-3352 online

http://www.jstage.jst.go.jp/browse/jos/ http://mc.manusriptcentral.com/jjocs
} 
multiphasic moods and comprises six mood scales classified into one positive (vigor) and five negative (tension-anxiety, depression-dejection, anger-hostility, fatigue, and confusion) moods ${ }^{17)}$. Most other tests only measure one aspect of mood, such as depression with the Self-rating Depression Scale and anxiety with the Manifest Anxiety Scale. POMS can be used to evaluate both patients and healthy people, and is standardized in various countries. Thus, POMS is considered suitable to multiphasically evaluate mood in healthy participants. Several intervention trials have evaluated the effect of DHA/EPA supplementation on mood in healthy adults ${ }^{18-20)}$ and elderly participants ${ }^{21}$ by using POMS, although no study regarding ARA supplementation has been reported. In healthy adults, Fontani et al. evaluated the effect of LCPUFA supplementation $(1.6 \mathrm{~g} /$ day EPA and $0.8 \mathrm{~g} /$ day DHA) for 5 weeks on the mood in healthy adults ${ }^{18,19)}$. EPA/DHA supplementation led to an increased score for the positive mood (vigor) and decreased scores for the five negative moods (tension-anxiety, depression-dejection, anger-hostility, fatigue, and confusion). In another study, supplementation with $1.7 \mathrm{~g} /$ day EPA and 0.3 g/day DHA for 4 weeks slightly improved fatigue ${ }^{20)}$. Regarding healthy elderly participants, only one study has evaluated the effect of DHA/EPA( 0.4 or $1.9 \mathrm{~g} /$ day) supplementation, and no obvious effects of LCPUFA on mood were observed $^{21)}$. These studies suggest that LCPUFA supplementation improves the mood in young and middle-aged healthy people. However, the effects of LCPUFA on the mood in healthy elderly people remains unknown.

Therefore, we hypothesized that LCPUFA supplementation improves mood in healthy elderly individuals. The objective of this study was to evaluate the effects of LCPUFA supplementation on mood in cognitively and emotionally normal elderly people. Previously, we conducted a randomized controlled trial(RCT) to evaluate the efficacy of LCPUFA (300 mg/day DHA, 100 mg/day EPA, and 120 mg/ day ARA) on cognitive function in Japanese elderly men ${ }^{22}$. In the present study, we examined the effect of this supplementation on POMS in elderly Japanese men by analyzing the secondary outcome from this previously performed $\mathrm{RCT}$ on cognitive function.

\section{EXPERIMENTAL}

\subsection{Study design and participants}

This study was performed by analyzing the secondary outcome in the previously performed randomized, doubleblind, placebo-controlled, parallel group intervention trial. This study was designed to evaluate the effects of LCPUFA supplementation on cognitive function in elderly Japanese men. The details of the participants and the study design, including inclusion and exclusion criteria for enrollment in this study, were described previously ${ }^{22}$. In brief, the par- ticipants were Japanese males aged 55-64 years without dementia or depression. Four hundred thirteen participants were screened, and 115 were randomly allocated to the placebo or LCPUFA group. Participants received capsules with purified olive oil (placebo) or LCPUFA-containing oil (including DHA, EPA, and ARA) for 4 weeks. The MiniMental State Examination and Beck Depression InventorySecond Edition were used for screening. Samples of blood and urine were obtained after an overnight fast. Physical and physiological parameters were measured, and the POMS was assessed at baseline and 4 weeks after the supplementation period. The fatty acid content in plasma phospholipids and dietary fatty acid intake was measured at baseline and 4 weeks after the supplementation period. The Ethics Committee on Human Experimentation of Suntory Holdings Ltd. approved the study protocol, which conformed to the principles set forth in the Declaration of Helsinki. Written informed consent was obtained from all participants before their entry to this study.

\subsection{Randomization and allocation}

As described previously ${ }^{22)}$, the enrolled participants were assigned in a 1:1 ratio to one of the two masked supplements, placebo or LCPUFA, based on a random number table. The randomization codes for these participants and the codes for the masked supplements were held by two different people not involved in this study, and information regarding these assignments was masked to researchers until all data were collected and analyzed.

\subsection{Experimental supplement}

The experimental supplements in this study were purified olive oil and LCPUFA-containing oil, the fatty acid compositions of which were analyzed based on AOCS official method Ce $1 \mathrm{~b}-89^{35}$ and shown in Table 1. In brief, LCPUFA-containing oil or purified olive oil extracted from capsules was incubated with $0.5 \mathrm{M} \mathrm{NaOH}$ methanol solution at $100^{\circ} \mathrm{C}$. After cooling, $\mathrm{BF}_{3} /$ methanol reagent was added to above solution and heated at $100^{\circ} \mathrm{C}$. Fatty acid methyl esters were extracted with $n$-hexane and analyzed using capillary gas-liquid chromatography. The LCPUFA group consumed 1,033 mg/day LCPUFA-containing oil in six soft gelatin capsules in which $300 \mathrm{mg}$ DHA, $100 \mathrm{mg}$ EPA, and $120 \mathrm{mg}$ ARA were included as free fatty acid equivalents. The same amount of purified olive oil was administered in the placebo group. The participants were asked to take six capsules after their first meal for 4 weeks.

\subsection{Outcome assessments}

The primary outcomes of this RCT have been described previously ${ }^{22}$. In brief, the primary outcome of this study was cognitive processing speed evaluated by event-related potential P300. The secondary outcomes were mood assessed by POMS, LCPUFA (DHA, EPA, and ARA) content in 
Table 1 Fatty acid composition of experimental supplements.

\begin{tabular}{lccc}
\hline FA & & $\begin{array}{c}\text { Placebo } \\
\text { (Purified olive oil) } \\
(\%)\end{array}$ & $\begin{array}{c}\text { LCPUFA- } \\
\text { containing oil } \\
(\%)\end{array}$ \\
\hline Palmitic acid & $16: 0$ & 13.6 & 6.4 \\
Stearic acid & $18: 0$ & 3.0 & 2.6 \\
Arachidic acid & $20: 0$ & 0.4 & 0.3 \\
Behenic acid & $22: 0$ & - & 0.9 \\
Lignoceric acid & $24: 0$ & - & 2.2 \\
Palmitoleic acid & $16: 1$ & 1.4 & 3.5 \\
Oleic acid & $18: 1$ & 68.1 & 11.7 \\
Eicosenoic acid & $20: 1$ & 0.2 & 1.4 \\
Docosenoic acid & $22: 1$ & - & 1.0 \\
Tetracosenoic acid & $24: 1$ & - & 0.6 \\
Linoleic acid & $18: 2 \mathrm{n}-6$ & 11.8 & 3.1 \\
Eicosadienoic acid & $20: 2 \mathrm{n}-6$ & - & 0.3 \\
Dihomo- $\gamma$-linolenic acid & $20: 3 \mathrm{n}-6$ & - & 0.9 \\
ARA & $20: 4 \mathrm{n}-6$ & - & 12.2 \\
Docosatetraenoic acid & $22: 4 \mathrm{n}-6$ & - & 0.3 \\
Docosapentaenoic acid & $22: 5 \mathrm{n}-6$ & - & 1.3 \\
$\alpha$-Linolenic acid & $18: 3 \mathrm{n}-3$ & 0.6 & 0.5 \\
EPA & $20: 5 \mathrm{n}-3$ & - & 9.7 \\
Docosapentaenoic acid & $22: 5 \mathrm{n}-3$ & - & 1.9 \\
DHA & $22: 6 \mathrm{n}-3$ & - & 29.8 \\
Others & & 0.9 & 9.4 \\
Total & & 100.0 & 100.0 \\
\hline LCP & & -9 \\
\hline
\end{tabular}

LCPUFA, long-chain polyunsaturated fatty acids; FA, fatty acid; ARA, arachidonic acid; EPA, eicosapentaenoic acid; DHA, docosahexaenoic acid.

plasma phospholipids, and dietary LCPUFA(DHA, EPA, and ARA) intake. A safety assessment was also performed, and it was previously reported that LCPUFA supplementation in the present study was considered safe under the conditions described here ${ }^{22)}$.

\subsection{Mood assessment with Profile of Mood States}

To report their mood over the preceding week, the participants were asked to fill out the Japanese edition of POMS, which comprises 65 questions $^{16,17)}$. POMS consists of six mood scales classified into positive mood (vigor)and negative mood (tension-anxiety, depression-dejection, anger-hostility, fatigue, and confusion) scales. The 65 questions in POMS include 58 questions for mood scales (vigor (8), tension-anxiety (9), depression-dejection (15), angerhostility (12), fatigue (7), and confusion(7)) and seven dummy questions. The participants selected from raw scores, which included five rating scores ranging from "not at all" (0) to "extremely" (4). These raw scores were summed to generate six mood scales. To use parametric statistical analysis, the raw scores for each scale were converted to a standard score (T-score) from an age- and sexdependent conversion table ${ }^{17}$. The scaling of possible scores (lowest to highest) of the T-score is: vigor (26-79); tension-anxiety (31-85); depression-dejection (40-85); anger-hostility (37-85); fatigue (38-85); and confusion (3185). A score of 50 corresponds to the average score of standard data that consider age and sex.

\subsection{Fatty acid analysis}

Blood was centrifuged at $1,470 \times g$ for $10 \mathrm{~min}$, and the plasma samples were stored at $-80^{\circ} \mathrm{C}$ prior to fatty acid analysis. Lipid extraction, purification, and fatty acid analysis were performed as described previously ${ }^{22}$. In brief, lipids in plasma were extracted and purified using the method of Folch et $a l .^{31)}$. Each lipid fraction was separated using thin-layer chromatography, and fatty acids in phospholipids were transmethylated. Fatty acid methyl esters 
were extracted and analyzed using capillary gas-liquid chromatography (Agilent 6890, Agilent Technologies, Santa Clara, CA, USA).

\subsection{Dietary assessment and study diary}

As described previously ${ }^{22}$, the dietary habits of the participants during the preceding month were assessed using the brief self-administered diet history questionnaire $(\mathrm{BDHQ})^{32)}$. Dietary intake including DHA, EPA, and ARA was estimated using an ad hoc computer algorithm for the BDHQ based on the Standard Tables of Food Composition in Japan, 2010. The participants were asked to keep a record in the study diary throughout the study.

\subsection{Statistical analysis}

To assess the effect of LCPUFA supplementation on mood, the exclusion criteria for statistical analysis were: a change in dietary LCPUFA intake between baseline and 4 weeks after supplementation (3 times larger than the amount of LCPUFA supplementation); an event affecting the mood, such as death of a relative, during the supplementation period. Data are shown as the mean \pm standard error (SE). Baseline data between the groups were compared with the unpaired t-test for quantitative variables or with the chi-square test or Mann-Whitney U test for qualitative variables. The change from baseline to 4 weeks after supplementation in each group was compared with the paired t-test. Comparisons of changes between groups were performed with the unpaired t-test. For all tests, twotailed $p$-values of less than 0.05 were considered to be statistically significant.

\section{RESULTS}

\subsection{Participant flow and characteristics}

The participant flow diagram is shown in Fig. 1. A total of 413 participants were screened; 115 participants were enrolled and randomly allocated to the placebo $(n=58)$ or LCPUFA $(n=57)$ group. One participant in each group withdrew consent before the supplementation. One hundred thirteen participants (placebo, $\mathrm{n}=57$; LCPUFA, $\mathrm{n}$ $=56)$ completed the 4 -week supplementation period, and 100 participants (placebo, $\mathrm{n}=49$; LCPUFA, $\mathrm{n}=51$ ) who were eligible for evaluation of mood were analyzed. Participants were excluded from the analysis for the following reasons: a change in dietary LCPUFA intake $(n=12)$ and experiencing an event that affected the $\operatorname{mood}(n=1)$. The baseline characteristics are shown in Table 2. The placebo and LCPUFA groups were matched for age, height, body weight, body mass index, education, occupation, alcohol consumption, smoking status, LCPUFA(DHA, EPA, and ARA) composition in plasma phospholipids, and LCPUFA intake from daily diets.
Assessed for eligibility $(n=413)$
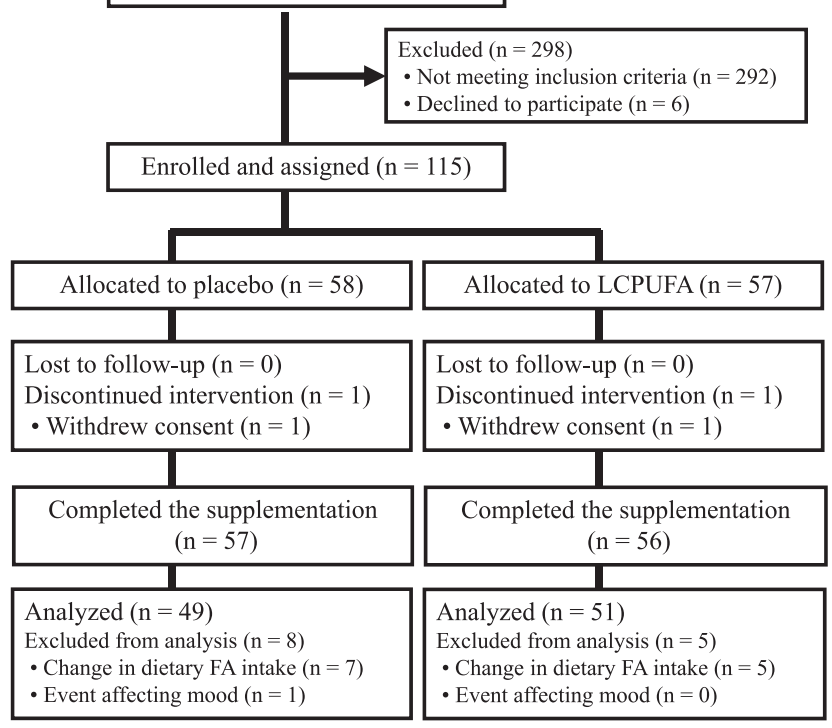

Fig. 1 Flow diagram of the study.

\subsection{Mood (Profile of Mood States)}

The six mood scales of POMS in both groups are shown in Fig. 2. The vigor scores, reflecting positive mood, at baseline in the placebo and the LCPUFA groups were 49.9 \pm 1.5 and $48.7 \pm 1.3$, respectively, with no significant difference between groups. The vigor score at 4 weeks in the LCPUFA group (Fig. 2B) was increased significantly by 1.8 ( $p=0.009 v s$. baseline), whereas that in the placebo group (Fig. 2A) was decreased by 0.5 compared with baseline, but not significantly. The change in vigor score after 4 weeks of supplementation (Fig. 2C) was significantly different between groups $(p=0.040)$. Regarding the other five scores reflecting negative mood scales (tension-anxiety, depression-dejection, anger-hostility, fatigue, and confusion), no significant differences were found between groups or between before and after supplementation.

\subsection{Fatty acid compositions in plasma phospholipids and fatty acid intake from daily diets}

Table 3 shows the LCPUFA composition in plasma phospholipids. The DHA, EPA, and ARA content at baseline was not different between the two groups. DHA and ARA compositions in the LCPUFA group at 4 weeks were increased significantly by $0.8 \%(p<0.001 v s$. baseline) and $0.7 \%(p<$ 0.001 vs. baseline), respectively. The DHA and ARA content in the placebo group remained unchanged during supplementation. Changes in the DHA and ARA content differed significantly between groups $(p=0.006$ and 0.002 , respectively). Regarding the EPA content in plasma, no significant differences were found between groups or between before and after supplementation.

Table 4 shows fatty acid intake from daily diets. No significant differences were found in DHA, EPA, or ARA 
Table 2 Baseline characteristics of participants.

\begin{tabular}{|c|c|c|c|}
\hline & Placebo group $(n=49)$ & LCPUFA group $(\mathrm{n}=51)$ & $p$ value \\
\hline Age $\left(\right.$ years) ${ }^{\mathrm{a}}$ & $59.7 \pm 0.4$ & $59.1 \pm 0.4$ & 0.315 \\
\hline Height $(\mathrm{cm})^{\mathrm{a}}$ & $169.3 \pm 0.8$ & $169.2 \pm 0.6$ & 0.965 \\
\hline Body weight $(\mathrm{kg})^{\mathrm{a}}$ & $66.3 \pm 1.3$ & $67.8 \pm 1.3$ & 0.409 \\
\hline $\mathrm{BMI}^{\mathrm{a}}$ & $23.1 \pm 0.4$ & $23.6 \pm 0.4$ & 0.317 \\
\hline Education $^{\mathrm{b}}$ & & & 0.126 \\
\hline Junior high & 0 & 0 & \\
\hline High & 12 & 8 & \\
\hline College & 6 & 2 & \\
\hline University & 31 & 41 & \\
\hline Occupation $^{\mathrm{b}}$ & & & 0.958 \\
\hline Full-time job & 34 & 34 & \\
\hline Part-time job & 8 & 9 & \\
\hline None & 7 & 8 & \\
\hline Alcohol consumption ${ }^{c}$ & & & 0.164 \\
\hline Daily & 11 & 7 & \\
\hline 1-6 days/week & 25 & 23 & \\
\hline 1-3 days/month & 3 & 10 & \\
\hline None & 10 & 11 & \\
\hline Smoking habit ${ }^{\mathrm{c}}$ & & & 0.673 \\
\hline$>19$ cigarettes/day & 6 & 4 & \\
\hline $1-19$ cigarettes/day & 5 & 6 & \\
\hline None & 38 & 41 & \\
\hline MMSE $^{a}$ & $29.6 \pm 0.2$ & $29.3 \pm 0.2$ & 0.112 \\
\hline BDI-II ${ }^{\mathrm{a}}$ & $4.5 \pm 0.6$ & $4.7 \pm 0.6$ & 0.769 \\
\hline \multicolumn{4}{|c|}{ FA composition in plasma PLs ${ }^{a}$} \\
\hline ARA $(\%)$ & $8.9 \pm 0.2$ & $8.6 \pm 0.2$ & 0.190 \\
\hline $\mathrm{EPA}(\%)$ & $2.3 \pm 0.2$ & $2.6 \pm 0.2$ & 0.417 \\
\hline DHA (\%) & $6.9 \pm 0.2$ & $7.0 \pm 0.2$ & 0.802 \\
\hline \multicolumn{4}{|l|}{ Dietary FA intake ${ }^{a}$} \\
\hline ARA (mg/day) & $150 \pm 10$ & $161 \pm 8$ & 0.363 \\
\hline EPA (mg/day) & $282 \pm 29$ & $259 \pm 23$ & 0.537 \\
\hline DHA (mg/day) & $477 \pm 46$ & $453 \pm 34$ & 0.671 \\
\hline
\end{tabular}

Mean \pm SE. We found no significant difference between the groups in baseline data ( ${ }^{a}$ unpaired t-test, ${ }^{b}$ chi-square test or ${ }^{c}$ Mann-Whitney U test).

LCPUFA, long-chain polyunsaturated fatty acids; BMI, body mass index; MMSE, Mini-Mental State Examination; BDI-II, Beck Depression Inventory-Second Edition; FA, fatty acid; PLs, phospholipids; ARA, arachidonic acid; EPA, eicosapentaenoic acid; DHA, docosahexaenoic acid.

intake between groups or between before and after supplementation. In addition, changes in intake of these fatty acids did not differ between groups.

\section{DISCUSSION}

In the present study, we examined the effect of LCPUFA (300 mg/day DHA, $100 \mathrm{mg} /$ day EPA, and $120 \mathrm{mg} /$ day ARA) on POMS in elderly Japanese men as the secondary outcome in a previously conducted RCT on cognitive function. The results showed that supplementation with 

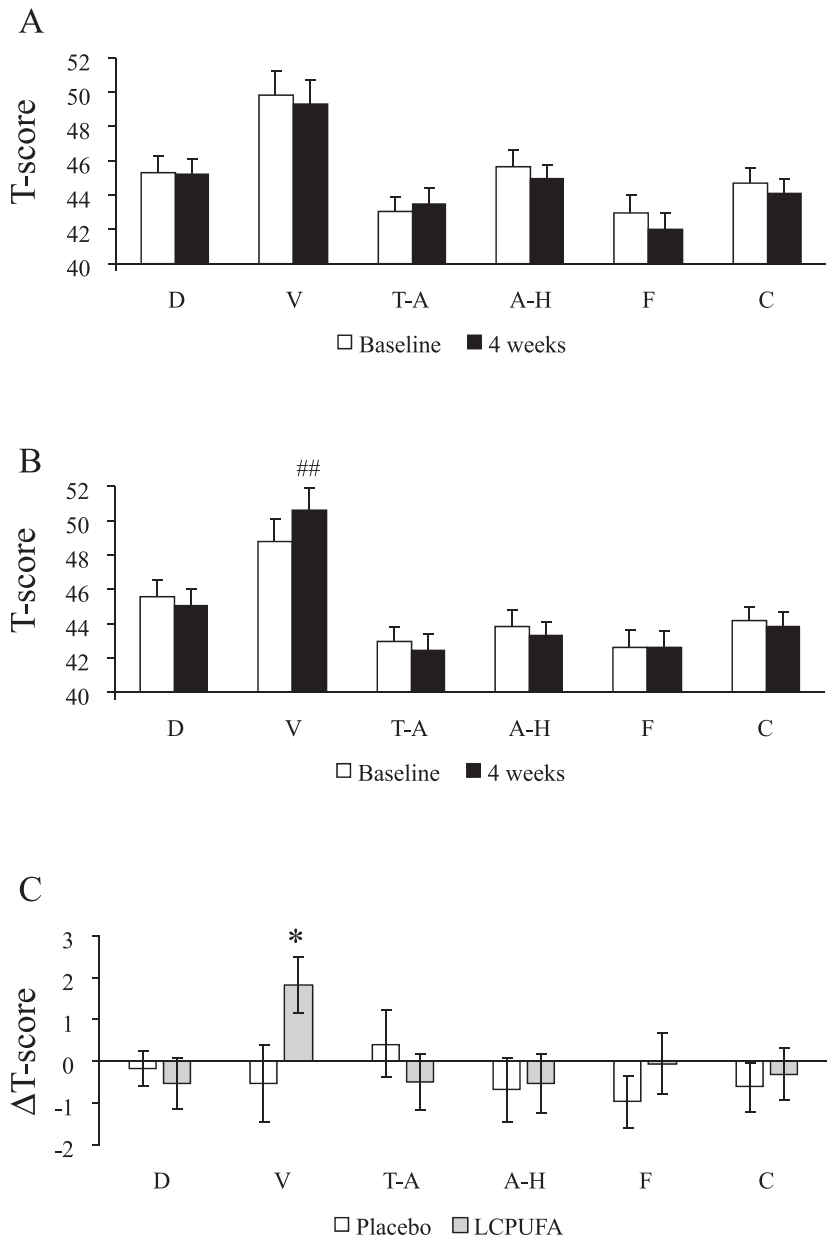

Fig. 2 Scores of POMS in the placebo and LCPUFA groups at baseline and 4 weeks after supplementation.

(A) Scores of POMS in the placebo group $(n=49)$ at baseline and 4 weeks after supplementation. We found no significant differences in the six moods between baseline and after supplementation (paired t-test). Mean \pm SE. (B) Scores of POMS in the LCPUFA group $(n=51)$ at baseline and 4 weeks after supplementation. \#\#p<0.01 vs. baseline (paired t-test). Mean \pm SE. (C) Changes in POMS after 4 weeks of supplementation in the placebo (n $=49)$ and LCPUFA $(\mathrm{n}=51)$ groups. ${ }^{*} p<0.05$ vs. the placebo group (unpaired t-test). Mean $\pm \mathrm{SE}$.

D: depression-dejection, V: vigor, T-A: tensionanxiety, A-H: anger-hostility, F: fatigue, C: confusion.

LCPUFA improved positive mood (vigor). This is the first report to suggest the efficacy of LCPUFA supplementation on mood in the elderly.

POMS comprises six mood scales classified into one positive (vigor) and five negative (tension-anxiety, depression-dejection, anger-hostility, fatigue, and confusion) moods. In the present study, the change in the vigor score for the LCPUFA group was significantly different from and 2.4 larger than that for the placebo group. The difference in vigor could be reliable because changes in all of the raw scores (eight questions) for vigor after 4-week supplementation in the LCPUFA group tended to be larger than those in the placebo group (data not shown). The difference of 2.4 was comparable to differences due to the effects of known inventions, such as $2.4 \mathrm{~g} /$ day EPA/DHA supplementation $(+4.2)^{19)}$ or forest therapy $(+4.4)^{3)}$ in healthy adults. These data suggest that the difference in vigor in the present study was psychologically meaningful, and that the efficacy of LCPUFA on vigor was observed regardless of age. On the other hand, the effects of LCPUFA on negative moods were not consistent with previous studies. Fontani et al. reported that $1.6 \mathrm{~g} /$ day EPA and $0.8 \mathrm{~g} /$ day DHA supplementation for 5 weeks not only increases vigor but also decreases tension-anxiety, depression-dejection, angerhostility, fatigue, and confusion in healthy adults ${ }^{18,19)}$. This inconsistency may be due to differences in the doses of LCPUFA or the characteristics of the study participants such as age and race. In addition, changes in the positive negative moods were not always linked in other intervention studies using foods ${ }^{23,24)}$. These data suggest that some interventions may independently affect positive and negative moods.

In a previous study in the elderly, DHA/EPA $(226 \mathrm{mg} /$ day DHA and 176 mg/day EPA or 1093 mg/day DHA and 843 $\mathrm{mg} /$ day EPA) supplementation for 26 weeks showed no obvious effects on mood ${ }^{21}$, although LCPUFA (300 mg/day DHA, $100 \mathrm{mg} /$ day EPA, and $120 \mathrm{mg}$ /day ARA) supplementation for 4 weeks was effective on the positive mood under our study conditions. Making a conclusion regarding the efficacy of LCPUFA on mood in healthy elderly people is currently difficult. However, the various results may be due to differences among these studies in terms of the characteristics of subjects such as race, the combination of DHA, EPA, and ARA, or the supplementation period. Therefore, further studies are necessary to clarify the efficacy of LCPUFA on mood in healthy elderly people.

Significant increases in DHA $(+0.8 \%)$ and ARA ( + $0.7 \%)$ content in plasma phospholipids were observed in the LCPUFA group, although no significant changes were observed in the placebo group. The increase in DHA and ARA content in plasma phospholipids is considered to be within a reasonable range, because the increase in DHA content was $3.2 \%$ with $900 \mathrm{mg} /$ day DHA supplementation $^{25)}$, and the increases in ARA content were $0.7 \%$ and $2.5 \%$ with $80 \mathrm{mg} /$ day and $240 \mathrm{mg}$ /day ARA supplementation, respectively ${ }^{26,27)}$. The EPA content in plasma phospholipids was likely not affected by the dose or the combination of LCPUFA (300 mg/day DHA, $100 \mathrm{mg} /$ day EPA, and $120 \mathrm{mg} /$ day ARA) supplementation in this study condition. On the other hand, we found no significant changes in 
Table 3 Fatty acid composition(\%) in plasma phospholipids in the placebo and LCPUFA groups during supplementation.

\begin{tabular}{|c|c|c|c|c|c|c|}
\hline \multirow{2}{*}{ FA } & \multicolumn{3}{|c|}{ Placebo group $(n=49)$} & \multicolumn{3}{|c|}{ LCPUFA group $(\mathrm{n}=51)$} \\
\hline & Baseline & 4 weeks & $\Delta$ & Baseline & 4 weeks & $\Delta$ \\
\hline PA & $27.3 \pm 0.2$ & $27.3 \pm 0.2$ & $0.0 \pm 0.1$ & $27.4 \pm 0.1$ & $27.2 \pm 0.1$ & $-0.2 \pm 0.1$ \\
\hline SA & $14.4 \pm 0.1$ & $14.4 \pm 0.1$ & $0.0 \pm 0.1$ & $14.4 \pm 0.1$ & $14.6 \pm 0.1$ & $0.2 \pm 0.1$ \\
\hline OA & $10.3 \pm 0.2$ & $10.9 \pm 0.2^{\#}$ & $0.6 \pm 0.2$ & $10.4 \pm 0.2$ & $10.5 \pm 0.2$ & $0.1 \pm 0.2$ \\
\hline LA & $21.3 \pm 0.4$ & $20.7 \pm 0.4$ & $-0.6 \pm 0.3$ & $20.8 \pm 0.4$ & $20.0 \pm 0.5^{\#}$ & $-0.8 \pm 0.4$ \\
\hline ARA & $8.9 \pm 0.2$ & $9.1 \pm 0.2$ & $0.2 \pm 0.1$ & $8.6 \pm 0.2$ & $9.3 \pm 0.2^{\# \#}$ & $0.7 \pm 0.1 * *$ \\
\hline $\mathrm{EPA}$ & $2.3 \pm 0.2$ & $2.5 \pm 0.2$ & $0.2 \pm 0.2$ & $2.6 \pm 0.2$ & $2.7 \pm 0.2$ & $0.1 \pm 0.2$ \\
\hline DHA & $6.9 \pm 0.2$ & $7.2 \pm 0.2$ & $0.2 \pm 0.1$ & $7.0 \pm 0.2$ & $7.8 \pm 0.2^{\# \#}$ & $0.8 \pm 0.2 * *$ \\
\hline
\end{tabular}

Mean \pm SE. We found no significant difference between the groups for each fatty acid at baseline (unpaired t-test). ${ }^{* *} p<0.01$ vs. the placebo group (unpaired t-test). ${ }^{*} p<0.05 v s$. baseline (paired t-test). ${ }^{\#} p<0.01 v s$. baseline (paired t-test).

LCPUFA, long-chain polyunsaturated fatty acids; FA, fatty acid; PA, palmitic acid; SA, stearic acid; OA, oleic acid; LA, linoleic acid; ARA, arachidonic acid; EPA, eicosapentaenoic acid; DHA, docosahexaenoic acid.

Table 4 Dietary fatty acid intake (mg/day) in the placebo and LCPUFA groups during supplementation.

\begin{tabular}{cccccccc}
\hline \multirow{2}{*}{ FA } & \multicolumn{3}{c}{ Placebo group $(\mathrm{n}=49)$} & & \multicolumn{3}{c}{ LCPUFA group $(\mathrm{n}=51)$} \\
\cline { 2 - 4 } \cline { 6 - 7 } & Baseline & 4 weeks & $\Delta$ & & Baseline & 4 weeks & $\Delta$ \\
\hline LA & $10,000 \pm 512$ & $9,490 \pm 552$ & $-533 \pm 297$ & & $10,600 \pm 411$ & $10,000 \pm 452^{\#}$ & $-636 \pm 309$ \\
ALA & $1,590 \pm 85$ & $1,500 \pm 94$ & $-90 \pm 55$ & & $1,670 \pm 67$ & $1,540 \pm 70^{\#}$ & $-129 \pm 51$ \\
ARA & $150 \pm 10$ & $142 \pm 10$ & $-7 \pm 5$ & & $161 \pm 8$ & $152 \pm 8$ & $-9 \pm 7$ \\
EPA & $282 \pm 29$ & $276 \pm 29$ & $-5 \pm 14$ & & $259 \pm 23$ & $249 \pm 22$ & $-10 \pm 17$ \\
DHA & $477 \pm 46$ & $466 \pm 46$ & $-11 \pm 21$ & & $453 \pm 34$ & $439 \pm 34$ & $-14 \pm 27$ \\
\hline
\end{tabular}

Mean \pm SE. We found no significant difference between the groups in each fatty acid at baseline (unpaired t-test). ${ }^{*} p$ $<0.05 v s$. baseline (paired t-test).

LCPUFA, long-chain polyunsaturated fatty acids; FA, fatty acid; LA, linoleic acid; ALA, $\alpha$-linolenic acid; ARA, arachidonic acid; EPA, eicosapentaenoic acid; DHA, docosahexaenoic acid.

DHA, EPA, or ARA intake from daily diets between baseline and 4 weeks after supplementation in either group. These data suggest that the increases in plasma LCPUFA were due to LCPUFA supplementation.

Regarding the individual contribution of each LCPUFA component, EPA seems to affect mood in previous studies. Supplementation with LCPUFA using a higher dose of EPA than DHA improves mood in healthy young and middleaged people ${ }^{18-20)}$. Moreover, EPA appears to ameliorate the symptoms of depression more than $\mathrm{DHA}^{28)}$. In contrast, the present study suggests the possibility that DHA and ARA may be more effective than EPA on mood in the elderly, because only DHA and ARA but not EPA in plasma phospholipids were increased in this study. The individual contributions of DHA, EPA, and ARA on efficacy in the elderly are still unclear. An interesting question has arisen, and further studies using DHA, EPA, and/or ARA are expected to clarify the effects of individual LCPUFA.

In previous studies, the effective dose of EPA/DHA for mood in healthy adults was more than $2 \mathrm{~g} /$ day $^{18-20)}$, which is higher than average dietary intake levels in various countries (EPA, 0.05-0.4 g/day and DHA, 0.1-0.6 g/day) ${ }^{29)}$. In the present study, the average amounts of DHA, EPA, and ARA intake from daily diets at baseline were $477 \mathrm{mg} /$ day, $282 \mathrm{mg} /$ day, and $150 \mathrm{mg} /$ day respectively, and were consistent with a previous report of Japanese elderly people(DHA, $\sim 620 \mathrm{mg} /$ day; EPA, $\sim 350 \mathrm{mg} /$ day; and ARA, $\sim 170 \mathrm{mg} /$ day ${ }^{30}{ }^{30}$. The LCPUFA intake in this study can be considered typical in Japanese elderly people. We also confirmed that the dose of LCPUFA supplementation in the present study was in the range of that from daily diets. These data suggest that low doses of LCPUFA supplementation corresponding to dietary intake levels may have the potential to positively affect mood in the elderly.

Our study has some limitations. First, the results of this study are based on the secondary outcome analysis of our previously performed $\mathrm{RCT}^{22)}$. To clarify the efficacy, confirmatory studies are needed. Next, the sample size in this 
study was not large and further larger scale studies are needed to make a general conclusion. Then, the participants were only male. Regarding gender, moods measured by POMS raw scores are not affected by gender in Japanese elderly people ${ }^{17)}$. In addition, blood DHA, EPA, and ARA levels in elderly men and women are similar ${ }^{30}$. These studies suggest that gender may have only a small effect in the present study, but further studies are necessary to clarify the effect of gender.

In conclusion, LCPUFA (300 mg/day DHA, 100 mg/day $\mathrm{EPA}$, and $120 \mathrm{mg} /$ day ARA) supplementation improved the positive mood (vigor) in elderly Japanese men in these study conditions. This is the first report to suggest the efficacy of LCPUFA supplementation on mood in the elderly. Further studies are expected to clarify the efficacy.

\section{ACKNOWLEDGEMENTS}

We thank laboratory members of Institute for Health Care Science for stimulating discussion and comments on this work.

\section{CONFLICT OF INTEREST}

This work was supported by Suntory Wellness Ltd. H. T., T. S., M. K., H. K., and H. S. are employees of Suntory Wellness Ltd., which markets health food products that include LCPUFA. Y. K. declares no conflict of interest regarding this study.

\section{AUTHOR CONTRIBUTIONS}

H.T. participated in the study design, acquired the data, analyzed the data, and drafted the manuscript. T.S. participated in acquisition and analysis of the data. H.K. participated in the study design, analyzed the data, and drafted the manuscript. H.S. participated in the study design and helped to interpret the findings. Y.K. participated in the study design, analyzed the data, and drafted and reviewed the manuscript.

\section{REFERENCES}

1) Cassilhas, R.C.; Viana, V.A.; Grassmann, V.; Santos, R.T.; Santos, R.F.; Tufik, S.; Mello, M.T. The impact of resistance exercise on the cognitive function of the elderly. Med. Sci. Sports Exerc. 39, 1401-1407 (2007).

2) Oda, S.; Matsumoto, T.; Nakagawa, K.; Moriya, K. Relaxation effects in humans of underwater exercise of moderate intensity. Eur. J. Appl. Physiol. Occup.
Physiol. 80, 253-259(1999).

3) Tsunetsugu, Y.; Park, B.J.; Lee, J.; Kagawa, T.; Miyazaki, Y. Psychological relaxation effect of forest therapy: results of field experiments in 19 forests in Japan involving 228 participants. Nihon Eiseigaku Zasshi 66, 670-676 (2011).

4) Hayasaka, S.; Tsutsumi, A.; Noda, T.; Murata, C.; Ojima, T. Effects of stone spa (Ganban-yoku) on psychological states. Complement. Ther. Clin. Pract. 151, 29-32 (2009).

5) Gorby, H.E.; Brownawell, A.M.; Falk, M.C. Do specific dietary constituents and supplements affect mental energy? Review of the evidence. Nutr. Rev. 68, 697718(2010).

6) Söderberg, M.; Edlund, C.; Kristensson, K.; Dallner, G. Fatty acid composition of brain phospholipids in aging and in Alzheimer's disease. Lipids 26, 421-425 (1991).

7) McNamara, R.K.; Liu, Y.; Jandacek, R.; Rider, T.; Tso, P. The aging human orbitofrontal cortex: decreasing polyunsaturated fatty acid composition and associated increases in lipogenic gene expression and stearoylCoA desaturase activity. Prostaglandins Leukot. Essent. Fatty Acids 78, 293-304 (2008).

8) Fukaya, T.; Gondaira, T.; Kashiyae, Y.; Kotani, S.; Ishikura, Y.; Fujikawa, S.; Kiso, Y.; Sakakibara, M. Arachidonic acid preserves hippocampal neuron membrane fluidity in senescent rats. Neurobiol. Aging 28, 11791186 (2007).

9) Suzuki, H.; Park, S.J.; Tamura, M.; Ando, S. Effect of the long-term feeding of dietary lipids on the learning ability, fatty acid composition of brain stem phospholipids and synaptic membrane fluidity in adult mice: a comparison of sardine oil diet with palm oil diet. Mech. Ageing Dev. 101, 119-128(1998).

10) Kotani, S.; Nakazawa, H.; Tokimasa, T.; Akimoto, K.; Kawashima, H.; Toyoda-Ono, Y.; Kiso, Y.; Okaichi, H.; Sakakibara, M. Synaptic plasticity preserved with arachidonic acid diet in aged rats. Neurosci. Res. 46, 453-461 (2003).

11) McGahon, B.; Clements, M.P.; Lynch, M.A. The ability of aged rats to sustain long-term potentiation is restored when the age-related decrease in membrane arachidonic acid concentration is reversed. Neuroscience 81, 9-16 (1997).

12) McGahon, B.M.; Martin, D.S.; Horrobin, D.F.; Lynch, M.A. Age-related changes in synaptic function: analysis of the effect of dietary supplementation with omega-3 fatty acids. Neuroscience 94, 305-314 (1999).

13) Tokuda, H.; Kontani, M.; Kawashima, H.; Kiso, Y.; Shibata, H.; Osumi, N. Differential effect of arachidonic acid and docosahexaenoic acid on age-related decreases in hippocampal neurogenesis. Neurosci. Res. 88, 58-66 (2014).

14) Kawakita, E.; Hashimoto, M.; Shido, O. Docosahexae- 


\section{Long-chain polyunsaturated fatty acid and mood}

noic acid promotes neurogenesis in vitro and in vivo. Neuroscience 139, 991-997 (2006).

15) Giles, G.E.; Mahoney, C.R.; Kanarek, R.B. Omega-3 fatty acids influence mood in healthy and depressed individuals. Nutr. Rev. 71, 727-741(2013).

16) McNair, D.M.; Lorr, M.; Droppleman, L.F. Profile of Mood States. Educational and Industrial Testing Service, San Diego (1992).

17) Yokoyama, K.; Araki, S. POMS Japanese Manual (in Japanese). Kaneko Syoboh, Tokyo (1994).

18) Fontani, G.; Corradeschi, F.; Felici, A.; Alfatti, F.; Migliorini, S.; Lodi, L. Cognitive and physiological effects of Omega-3 polyunsaturated fatty acid supplementation in healthy subjects. Eur. J. Clin. Invest. 35, 691699 (2005).

19) Fontani, G.; Corradeschi, F.; Felici, A.; Alfatti, F.; Bugarini, R.; Fiaschi, A.I.; Cerretani, D.; Montorfano, G.; Rizzo, A.M.; Berra, B. Blood profiles, body fat and mood state in healthy subjects on different diets supplemented with Omega-3 polyunsaturated fatty acids. Eur. J. Clin. Invest. 35, 499-507(2005).

20) Antypa, N.; Van der Does, A.J.; Smelt, A.H.; Rogers, R.D. Omega-3 fatty acids (fish-oil) and depression-related cognition in healthy volunteers. J. Psychopharmacol. 23, 831-840 (2009).

21) van de Rest, O.; Geleijnse, J.M.; Kok, F.J.; van Staveren, W.A.; Hoefnagels, W.H.; Beekman, A.T.; de Groot, L.C. Effect of fish-oil supplementation on mental well-being in older subjects: a randomized, doubleblind, placebo-controlled trial. Am. J. Clin. Nutr. 88, 706-713 (2008).

22) Tokuda, H.; Sueyasu, T.; Kontani, M.; Kawashima, H.; Shibata, H.; Koga, Y. Low doses of long-chain polyunsaturated fatty acids affect cognitive function in elderly Japanese men: A randomized controlled trial. $J$. Oleo Sci. 64, 633-644(2015).

23) Kuroda, M.; Ishizaki, T.; Maruyama, T.; Takatsuka, Y.; Kuboki, T. Effect of dried-bonito broth on mental fatigue and mental task performance in subjects with a high fatigue score. Physiol. Behav. 92, 957-962 (2007).

24) Ussher, J.M.; Dewberry, C.; Malson, H.; Noakes, J. The relationship between health related quality of life and dietary supplementation in british middle managers: A double blind placebo controlled study. Psychol. Health 10, 97-111 (1995).

25) Yurko-Mauro, K.; McCarthy, D.; Rom, D.; Nelson, E.B.; Ryan, A.S.; Blackwell, A.; Salem, N,Jr.; Stedman, M.; MIDAS Investigators. Beneficial effects of docosahexaenoic acid on cognition in age-related cognitive decline. Alzheimer's. Dement. 6, 456-464(2010).

26) Kakutani, S.; Ishikura, Y.; Tateishi, N.; Horikawa, C.; Tokuda, H.; Kontani, M.; Kawashima, H.; Sakakibara, Y.; Kiso, Y.; Shibata, H.; Morita, I. Supplementation of arachidonic acid-enriched oil increases arachidonic acid contents in plasma phospholipids, but does not increase their metabolites and clinical parameters in Japanese healthy elderly individuals: a randomized controlled study. Lipids Health Dis. 10, 241 (2011).

27) Hirota, S.; Adachi, N.; Gomyo, T.; Kawashima, H.; Kiso, Y.; Kawabata, T. Low-dose arachidonic acid intake increases erythrocytes and plasma arachidonic acid in young women. Prostaglandins Leukot. Essent. Fatty Acids 83, 83-88(2010).

28) Martins, J.G. EPA but not DHA appears to be responsible for the efficacy of omega-3 long chain polyunsaturated fatty acid supplementation in depression: evidence from a meta-analysis of randomized controlled trials. J. Am. Coll. Nutr. 28, 525-542 (2009).

29) Elmadfa, I.; Kornsteiner, M. Fats and fatty acid requirements for adults. Ann. Nutr. Metab. 55, 56-75 (2009).

30) Otsuka, R.; Kato, Y.; Imai, T.; Ando, F.; Shimokata, H. Higher serum EPA or DHA, and lower ARA compositions with age independent fatty acid intake in Japanese aged 40 to 79. Lipids 48, 719-727(2013).

31) Folch, J.; Lees, M.; Sloane Stanley, G.H. A simple method for the isolation and purification of total lipids from animal tissues. J. Biol. Chem. 226, 497-509 (1957).

32) Murakami, K.; Mizoue, T.; Sasaki, S.; Ohta, M.; Sato, M.; Matsushita, Y.; Mishima, N. Dietary intake of folate, other B vitamins, and omega-3 polyunsaturated fatty acids in relation to depressive symptoms in Japanese adults. Nutrition 24, 140-147 (2008).

33) Marventano, S.; Kolacz, P.; Castellano, S.; Galvano, F.; Buscemi, S.; Mistretta, A.; Grosso, G. A review of recent evidence in human studies of n-3 and n-6 PUFA intake on cardiovascular disease, cancer, and depressive disorders: does the ratio really matter? Int. J. Food Sci. Nutr. 66, 611-22(2015).

34) Grosso, G.; Micek, A.; Marventano, S.; Castellano, S.; Mistretta, A.; Pajak, A.; Galvano, F. Dietary n-3 PUFA, fish consumption and depression: A systematic review and meta-analysis of observational studies. J. Affect. Disord. 205, 269-281 (2016).

35) American Oil Chemists' Society (AOCS). Fatty Acids Composition of Marine Oils, Official Method Ce 1b-89 (2009). 